The thiouracil-cytosine linkage does, however, have small effects on the biological activity of the tRNA which is shown in a reduced affinity for the synthetase and a slower rate of incorporation of valine from the charged tRNA into polypeptides during protein synthesis.

The photoproducts so far isolated from DNA never contain more than two pyrimidines linked together. Wang, however, has isolated and characterized trimers and tetramers from irradiation of pyrimidines in ice matrices and in water (J. Amer. Chem. Soc., 93, 2554 and $2768 ; 1971$ ) which could prove to have biological counterparts. The trimer in particular has the interesting property of reverting either to thymine and thymine dimer or to thymine and a thymine-adduct.

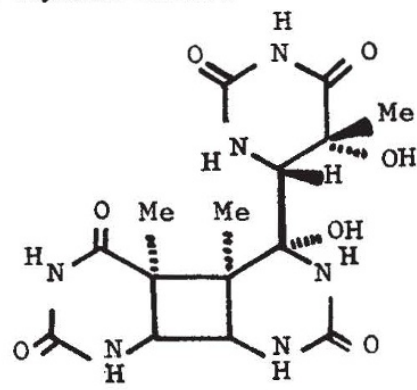

I

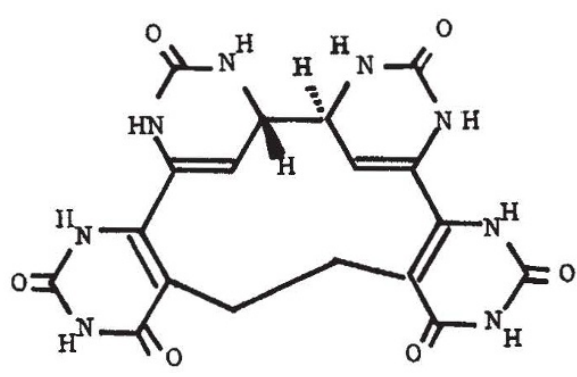

II

The structure (I) of the trimer and that of the tetramer (II), produced on irradiation of a thymine-uracil photoproduct, have been elucidated by Flippen and Karle (J. Amer. Chem. Soc., 93, 2556 and 2762 ; 1971) by Xray diffraction.

\section{ANTIBODIES}

\section{Garnering $\gamma$ Globulin}

from our Molecular Biology Correspondent A $6 \AA$ electron density map of an immunoglobulin has now been achieved by Davies and his colleagues (Sarma et al., J. Biol. Chem., 246, 3753 ; 1971) and is in gratifying accord with what has been deduced about the shape of the molecule by electron microscopy and other methods. In the crystal the molecules have a dyad symmetry axis, and the electron density distribution is interpretable in terms of three globular

elements, joined together so as to form a T-shape, the stem consisting of the so-called Fc portion which is attached by means of a rather narrow hinge to the two Fab components. The hinge gives access to proteolytic enzymes, which cleave at this point, and is surmised from a variety of lines of evidence to be flexible when the molecule is in solution. The size and shape of the Fc unit are in good agreement with data derived from a study of the crystals of this fragment alone from Poljak's laboratory.

In a companion article, Lebaw and Davies (ibid., 3760) show electron micrographs of the immunoglobulin crystal. They utilize the magic of optical integration-whereby multiple prints of the micrographs are made, advancing the photographic paper each time under the enlarger by a distance correspond. ing to the periodicity. The picture of a well-dug field then gradually resolves itself into a sharply-defined pattern, in which the repeating unit stands out as a $T$ or $Y$ shape, somewhat smaller than the hydrated molecules observed by $\mathrm{X}$-ray diffraction in the unfixed crystal,

\title{
The Truth ahout Juan de Fuca
}

THE appealing simplicity of seafloor spreading is probably an illusion on two counts. First, it would be surprising if a conceptual model so simple were to be rigorously applicable to a real Earth which is manifestly so complex, although the seafloor spreading model in its most basic form has done much to unify the Earth sciences. Current ideas on spreading will in the course of time no doubt come to be seen as a first approximation rather than as a detailed hypothesis for the behaviour of the Earth. But second, and perhaps more obviously, even if the seafloor is being, and has been, formed in a simple way, the final picture is likely to be quite complex. For notwithstanding the comparative youth of the oceans, changes may have taken place in the structure of the ocean floor since spreading began. Indeed, considering the state of activity in the oceanic crust it would be surprising if they had not.

As Peter and DeWald show in next Monday's Nature Physical Science, a good example of the latter type of complexity is the ocean floor off the northwest coast of the United States. Here the Juan de Fuca, Gorda and Explorer Ridges, each of which is offset from the others and each of which trends in a north-easterly direction, were clearly formed as a single rift but have since been subject to numerous fractures. The first job is thus to try to construct an authentic map of the anomalies and faults in the region; and this is what but exactly like the isolated molecules observed in the electron microscope by Valentine and Green some years ago.

These $\gamma \mathrm{G}$-immunoglobulin molecules consist of two heavy and two light chains, joined together by disulphide bonds, but other forms exist with a higher level of complexity. Thus electron microscopy has shown macroglobulin (immunoglobulin $\mathbf{M}$ ) to consist of a cyclic pentamer of $\gamma$ G-like (7S) subunits. The units are joined by disulphide bonds between their $\mathrm{Fc}$ regions. The determination of the sequence of events in the assembly, first of the four-chain structure, then of the pentameric form, has been one of the recent triumphs of the field, and some details of the mechanism are described in the latest article by Askonas and Parkhouse (Biochem. J., 123, 629; 1971). They showed previously that inside the tumour cells, in which the macroglobulin is made, only the $7 \mathrm{~S}$ subunits are present. By the time the newly synthesized protein is secreted assembly to the complete pentamers has occurred. When the subunits from lysed cells are allowed to stand in air, no pentamers

Peter and DeWald have done, partly with the help of data previously published and partly with thirteen new east-west magnetic traverses carried out off the coasts of Washington and Oregon.

Examination of the pattern of magnetic offsets clearly shows the existence of a previously undiscovered fracture zone separating the mountainous ridge topography from the Tufts Abyssal Plain. This Peter and DeWald have termed, appropriately, the Juan de Fuca Fracture Zone. But, more importantly, the discovery of the new fracture taken in conjunction with the complete anomaly map has enabled Peter and DeWald to end the speculation about why the original ridge broke up and why its component parts now trend north-east. The fact is that some of the anomalies west of the Juan de Fuca Ridge are missing and obviously disappear along the newly discovered fracture zone which coincides with a 550 metre change in seafloor relief. This must indicate deformation involving rotation, strike-slip faulting and the over thrusting of small crustal plates; and according to Peter and DeWald the most likely cause is compression in the roughly north-east-south-west direction. What is now quite clear is that the ridge trends in this region cannot be attributable to anything as gradual as a slow realignment produced by a change in the direction of seafloor spreading. 